\title{
AN EFFICIENT AND ROBUST METHOD FOR PREDICTING HELICOPTER ROTOR HIGH-SPEED IMPULSIVE NOISE
}

\author{
Kenneth S. Brentner* \\ NASA Langley Research Center \\ Hampton, Virginia
}

\begin{abstract}
A new formulation for the Ffowcs WilliamsHawkings quadrupole source, which is valid for a far-field in-plane observer, is presented. The far-field approximation is new and unique in that no further approximation of the quadrupole source strength is made and integrands with $r^{-2}$ and $r^{-3}$ dependence are retained. This paper focuses on the development of a retarded-time formulation in which time derivatives are analytically taken inside the integrals to avoid unnecessary computational work when the observer moves with the rotor. The new quadrupole formulation is similar to Farassat's thickness and loading formulation 1A. Quadrupole noise prediction is carried out in two parts: a preprocessing stage in which the previously computed flow field is integrated in the direction normal to the rotor disk, and a noise computation stage in which quadrupole surface integrals are evaluated for a particular observer position. Preliminary predictions for hover and forward flight agree well with experimental data. The method is robust and requires computer resources comparable to thickness and loading noise prediction.
\end{abstract}

\section{Notation}

$\square^{2} \quad$ wave operator

$c \quad$ sound speed in undisturbed medium

$d \Omega \quad$ element of the collapsing-sphere surface

$d S \quad$ element of the rotor-blade surface

$f=0 \quad$ function that describes the rotor-blade surface

$f^{+}=0$ surface described by union of rotor-blade surface and rotor disk

*Research Engineer, Aerodynamic and Acoustics Methods Branch, Fluid Mechanics and Acoustics Division, Senior Member AIAA.

Copyright (C) 1996 by the American Institute of Aeronautics and Astronautics, Inc. No copyright is asserted in the United States under Title 17, U.S. Code. The U.S. Government has a royalty-free license to exercise all rights under the copyright claimed herein for government purposes. All other rights are reserved by the copyright owner. $g=0 \quad$ surface that describes the collapsing sphere, $g=\tau-t+r / c$

$H(f)$ Heaviside function

$l_{i} \quad$ local force that acts on body

$\vec{M} \quad$ local velocity vector of source normalized by $c$, with components $M_{i}$

$\dot{M}_{i} \quad \partial M_{i} / \partial \tau$

$M_{r} \quad$ Mach number of source in radiation direction, $M_{i} \hat{r}_{i}$

$\dot{M}_{r} \quad \dot{M}_{i} \hat{r}_{i}$

$\ddot{M}_{r} \quad \ddot{M}_{i} \hat{r}_{i}$

$M_{A T} \quad$ advancing tip Mach number

$M_{H} \quad$ hover tip Mach number

$\hat{n} \quad$ unit outward normal vector to surface, with components $\hat{n}_{i}$

$p^{\prime} \quad$ acoustic pressure, $p-p_{0}$

$Q_{i j} \quad$ quadrupole surface source tensor, symmetric

$Q_{M M} \quad Q_{i j} M_{i} M_{j}$

$Q_{M r} \quad Q_{i j} M_{i} \hat{r}_{j}$

$Q_{\dot{M} r} \quad Q_{i j} \dot{M}_{i} \hat{r}_{j}$

$Q_{r r} \quad Q_{i j} \hat{r}_{i} \hat{r}_{j}$

$\dot{Q}_{M r} \quad \dot{Q}_{i j} M_{i} \hat{r}_{j}$

$\dot{Q}_{r r} \quad \dot{Q}_{i j} \hat{r}_{i} \hat{r}_{j}$

$\ddot{Q}_{r r} \quad \ddot{Q}_{i j} \hat{r}_{i} \hat{r}_{j}$

$r \quad$ distance between observer and source, $r=|\vec{x}-\vec{y}|$

$\hat{r} \quad$ unit vector in the radiation direction, with components $\hat{r}_{i}$

$R \quad$ rotor radii

$t \quad$ observer time

$T_{i j} \quad$ Lighthill stress tensor, $\rho u_{i} u_{j}+\left(p^{\prime}-c_{o}^{2} \rho^{\prime}\right) \delta_{i j}$ (inviscid form)

$v_{n} \quad$ local normal velocity of source surface

$\vec{x} \quad$ observer position vector, with components $x_{i}$

$\vec{y} \quad$ source position vector, with components $y_{i}$

Greek symbols:

$\Gamma \quad$ intersection of collapsing sphere $g=0$, and source surface $f=0$

$\delta(f) \quad$ Dirac delta function

$\theta \quad$ angle between $\hat{n}$ and $\hat{r}$

$\rho_{0} \quad$ density of undisturbed medium 
$\tau \quad$ source time

Subscript:

ret quantity is evaluated at the retarded time, $\tau=t-r / c$

\section{Introduction}

High-speed impulsive (HSI) noise is a particularly intense and annoying noise generated by helicopter rotors in high-speed forward flight. This HSI noise is closely associated with the appearance of shocks and transonic flow around the advancing rotor blades. The quadrupole sources in the Ffowcs WilliamsHawkings ( FW-H) equation ${ }^{1}$ account for nonlinearities in the vicinity of the rotor blade. These nonlinearities are of two types, which are described by Lighthill. $^{2,3}$ First, the local speed of sound is not constant but varies due to particle acceleration. Second, the finite particle velocity near the blade influences the velocity of sound propagation. By inclusion of the quadrupole source, the correct physics is mathematically simulated in the acoustic analogy. The quadrupole source in the $\mathrm{FW}-\mathrm{H}$ equation was identified by $\mathrm{Yu}$ et al. ${ }^{4}$ as a significant contributor to helicopter HSI noise. Hanson and Fink ${ }^{5}$ also included the quadrupole source for high-speed propeller noise prediction but found that it was not a significant noise source in that application. Even though this early work demonstrated the importance of the $\mathrm{FW}-\mathrm{H}$ quadrupole, it has not been routinely included in rotor noise predictions because of the difficulty in predicting the source strength of the Lighthill stress tensor $T_{i j}$ and the lack of a computationally efficient algorithm for computing the quadrupole noise.

In the past few years, the computation of the transonic aerodynamic field around rotor blades has become feasible; hence, renewed interest in prediction of HSI noise has emerged. Yu et al. ${ }^{4}$ were the first to successfully utilize advances in CFD by approximating the quadrupole source strength and integrating in the direction normal to the rotor plane. The integration in the normal direction of the approximate quadrupole source, which is valid in the far field ahead of the helicopter, effectively transforms the volume integration of the quadrupole into a surface integration. More recently, Schultz and Splettstoesser, ${ }^{6}$ Schultz et al., ${ }^{7}$ and Ianniello and De Bernardis ${ }^{8}$ have also used this technique with good results. Prieur ${ }^{9}$ and Prieur et al. ${ }^{10}$ have developed a frequency domain method for computing the quadrupole noise of hovering rotors that has yielded good results.

Some attempts have been made to numerically integrate the entire volume around the blade, 7,8 but the computations generally require computer resources comparable to those required by unsteady three-dimensional computational fluid dynamics (CFD) calculations - significantly more than that required for thickness and loading noise predictions. Farassat ${ }^{11}$ and his colleagues ${ }^{12,13}$ also tried to reduce the computational effort required in computing HSI noise; they recognized that the appearance of a shock wave coincides with the onset of HSI noise. By assuming that the shock is the dominant contributor of quadrupole noise, the acoustic sources are mathematically confined to the shock surface. When the shock-noise theory was implemented, the conclusion that the shock noise was a dominant component of the quadrupole source was verified. ${ }^{13}$ Nevertheless, the difficulty in accurately extracting the shock geometry, location, and strength from CFD solutions, together with the fact that the shock noise alone did not sufficiently characterize the total quadrupole source contribution, has postponed the complete implementation of the theory.

The goal of this work is to utilize the far-field approximation to the $\mathrm{FW}-\mathrm{H}$ quadrupole given by Brentner and Holland ${ }^{14}$ and extend the formulation to include forward-flight computations. This new formulation yields efficient numerical prediction of HSI noise without resorting to unnecessary or ad hoc simplifications of the $\mathrm{FW}-\mathrm{H}$ quadrupole source term. The mathematical manipulations used in this approach are rigorous and depend only on the far-field assumption, without approximation of the source strength. Numerical time differentiation of integrals is avoided throught the development of an alternate formulation in which the time differentiation is done analytically. Preliminary calculations with this new formulation demonstrate the potential for efficiency and robustness.

The acoustic analogy approach was chosen because of the substantial knowledge base gained in the development and utilization of thickness and loading noise predictions, based on the $\mathrm{FW}-\mathrm{H}$ equation. Further, the fundamental far-field assumption, which is described in the next section, leads to integrals of precisely the same form as current thickness and loading noise calculations; hence, the existing numerical algorithms can be used directly. Finally, the identification of individual noise components is a unique advantage of the acoustic analogy approach. This new formulation has been coded and is described in the remainder of this paper. The numerical results are compared with experimental data for both hover and forward-flight conditions. 


\section{Formulation Development}

The FW-H equation is the most general form of the Lighthill acoustic analogy and is appropriate for predicting the noise generated by the complex motion of helicopter rotors. In differential form, the $\mathrm{FW}-\mathrm{H}$ equation is given by the following inhomogeneous wave equation:

$$
\begin{aligned}
\square^{2} p^{\prime}(\vec{x}, t) & =\frac{\partial}{\partial t}\left[\rho_{o} v_{n} \delta(f)\right]-\frac{\partial}{\partial x_{i}}\left[l_{i} \delta(f)\right] \\
& +\frac{\partial^{2}}{\partial x_{i} \partial x_{j}}\left[T_{i j} H(f)\right]
\end{aligned}
$$

where $p^{\prime}(\vec{x}, t)$ is the acoustic pressure and the three source terms on the right-hand side are known as the thickness, loading, and quadrupole source terms, respectively. The rotor-blade surface is defined by the equation $f=0$. Note in equation (1) that the thickness and loading source terms are surface distributions of sources (indicated by the presence of the Dirac delta function $\delta(f))$. Also note that the quadrupole source is a volume distribution of sources (indicated by the Heaviside function $H(f)$ ). The $\mathrm{FW}-\mathrm{H}$ equation is valid in the entire unbounded space; hence, a formal solution may be obtained by using the free-space Green's function $\delta(g) / 4 \pi r$. In this paper, we are primarily concerned with the contribution of the quadrupole source term.

Farassat and Brentner ${ }^{15}$ have shown that after some manipulation of the formal solution the noise contribution from the quadrupole may be expressed as

$$
\begin{aligned}
4 \pi p_{Q}^{\prime}(\vec{x}, t) & =\frac{1}{c} \frac{\partial^{2}}{\partial t^{2}} \int_{-\infty}^{t} \int_{j>0} \frac{T_{r r}}{r} d \Omega d \tau \\
& +\frac{\partial}{\partial t} \int_{-\infty}^{t} \int_{f>0} \frac{3 T_{r r}-T_{i i}}{r^{2}} d \Omega d \tau(2) \\
& +c \int_{-\infty}^{t} \int_{f>0} \frac{3 T_{r r}-T_{i i}}{r^{3}} d \Omega d \tau
\end{aligned}
$$

where $p_{Q}^{\prime}(\vec{x}, t)$ is the acoustic pressure due to the quadrupole source. The quantity $T_{r r}$ is the double contraction $T_{i j} \hat{r}_{i} \hat{r}_{j}$, and $\hat{r}_{i}$ are the components of the unit vector in the radiation direction. In addition, $d \Omega$ is an element of the surface $g=0$, which is known as the collapsing sphere; hence, equation (2) is known as a collapsing-sphere formulation.

\section{Formulation Q1}

Equation (2) is the starting point for deriving the formulation developed by Brentner and Holland. ${ }^{14}$

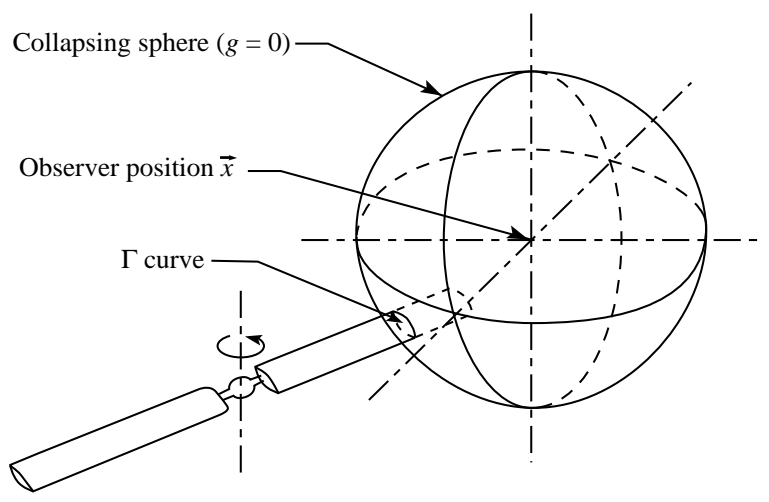

Figure 1. Schematic of collapsing sphere that intersects rotor blade.

To better illustrate the far-field approximation used in reference 14, we first give a geometric interpretation of the collapsing-sphere formulation. The collapsing sphere is defined by the equation $g=$ $\tau-t+r / c=0$, where $\tau$ and $t$ are the source and observer times, respectively, and $r$ is the distance between the observer position $\vec{x}$ and the source position $\vec{y}$. Because the observer time $t$ and the observer position $\vec{x}$ are held fixed during the integration, the solution to $g=0$ can be interpreted as a sphere centered on the observer $\vec{x}$ of radius $r=|\vec{x}-\vec{y}|$, which reduces, or collapses, as $\tau$ approaches $t$. A schematic is shown in figure 1.

An integration over the entire collapsing-sphere surface is not necessary in the integrals of equation (2) because the Lighthill stress tensor $T_{i j}$ vanishes away from the source region. For an observer in the far field, the collapsing sphere can be locally approximated by a cylinder, as shown in figure 2, because the source strength reduces quickly in the direction normal to the rotor blade. A further simplification in acoustic calculations occurs if the observer is assumed to be in the rotor plane (precisely where HSI noise has maximum directivity) because in this case integration in the direction normal to the rotor plane can be done independently of the observer position. $\mathrm{Yu}$ et al. ${ }^{4}$ were the first to use this far-field approximation for the evaluation of helicopter rotor HSI noise; however, the present work differs in that no additional approximation is made to the quadrupole source strength.

The integration over the approximate collapsingsphere surface is carried out in two stages. First, integration in the direction normal to the rotor disk is accomplished. This step allows us to define the 


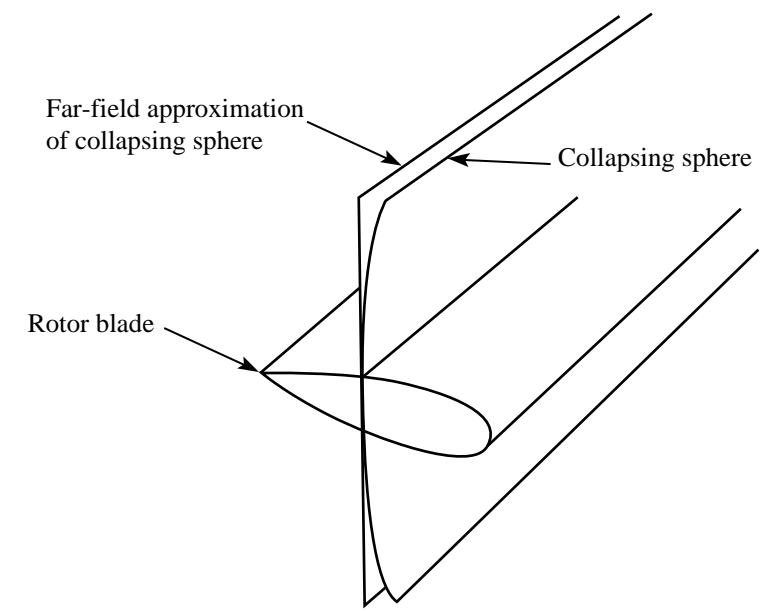

Figure 2. Schematic of approximation to collapsing sphere in source region.

quadrupole source strength on the rotor disk as

$$
Q_{i j}=\int_{j>0} T_{i j} d z
$$

where $z$ is understood to be in the direction normal to the rotor disk and the $z$ integration is only done outside the rotor blade. The new tensor $Q_{i j}$ is distributed on the rotor disk plane and is expected to vanish sufficiently far ahead of the leading edge, behind the trailing edge, and off the blade tip. By using relation (3), equation (2) can now be written as

$$
\begin{aligned}
& 4 \pi p_{Q}^{\prime}(\vec{x}, t)=\frac{1}{c} \frac{\partial^{2}}{\partial t^{2}} \int_{-\infty}^{t} \int_{\substack{f^{+}=0 \\
g=0}} \frac{Q_{r r}}{r} d \Gamma d \tau \\
& +\frac{\partial}{\partial t} \int_{-\infty}^{t} \int_{\substack{f^{+}=0 \\
g=0}} \frac{3 Q_{r r}-Q_{i i}}{r^{2}} d \Gamma d \tau \\
& +c \int_{-\infty}^{t} \int_{\substack{f^{+}=0 \\
g=0}} \frac{3 Q_{r r}-Q_{i i}}{r^{3}} d \Gamma d \tau
\end{aligned}
$$

where $f^{+}=0$ represents the rotor disk plane, including the blade surface. The intersection of the collapsing sphere with the rotor plane results in a curve for which we use the notation $\Gamma$. (See figure 1.)

The integrals in equation (4) are in the same form as the collapsing-sphere formulation for thickness and loading noise developed by Farassat. ${ }^{16}$ For this reason, we can apply the relation ${ }^{16}$

$$
\frac{c d \Gamma d \tau}{\sin \theta}=\frac{d S}{\left|1-M_{r}\right|}
$$

to transform equation (4) from a collapsing-sphere formulation to a retarded-time formulation. In equation (5), $d S$ is an element of the source surface, and $\theta$ is the angle between the source surface normal and the radiation direction. When the observer is in the rotor plane, $\sin \theta=1$. This transformation is exact.

The retarded-time formulation that results from the application of equation (5) to equation (4), first presented in reference 14 , is

$$
\begin{aligned}
4 \pi p_{Q}^{\prime}(\vec{x}, t) & =\frac{1}{c^{2}} \frac{\partial^{2}}{\partial t^{2}} \int_{f+=0}\left[\frac{Q_{r r}}{r\left|1-M_{r}\right|}\right]_{r e t} d S \\
& +\frac{1}{c} \frac{\partial}{\partial t} \int_{f^{+=0}}\left[\frac{3 Q_{r r}-Q_{i i}}{r^{2}\left|1-M_{r}\right|}\right]_{r e t} d S \\
& +\int_{f+=0}\left[\frac{3 Q_{r r}-Q_{i i}}{r^{3}\left|1-M_{r}\right|}\right]_{r e t} d S
\end{aligned}
$$

where $M_{r}$ is the local Mach number of the source in the radiation direction and the subscript ret indicates that the integrand is evaluated at the retarded time $t-r / c$. For convenience, we refer to this equation as formulation Q1. (This notation parallels that used by Farassat for the thickness and loading formulation 1.) Although formulation Q1 is strictly valid only in the rotor plane where $\sin \theta=1$, this formulation is expected to provide useful results for observers located at small angles above or below the rotor plane. For larger angles, the quadrupole contribution is not expected to be significant. In addition, because the far-field approximation only relies on the source strength vanishing in the direction normal to the rotor, the observer can be relatively close to the rotor (e.g., within 1 to 2 rotor radii).

Evaluation of the quadrupole noise can now be completed with substantially less computational effort than a direct numerical evaluation of either equation (2) or its retarded-time counterpart. Because the integrals in equation (6) are of the same form as thickness and loading noise, formulation Q1 can be easily incorporated into existing rotornoise prediction codes such as WOPWOP. ${ }^{17}$ Indeed, this formulation has been incorporated into WOPWOP by Brentner and Holland. ${ }^{14}$ Furthermore, the numerical insight, experience, robustness, and efficiency that has been realized in previous research can now be applied directly to the approximate quadrupole prediction. 


\section{Formulation Q1A}

One drawback of equation (6) is that numerical time differentiation of the first two integrals is required. If the observer is stationary, then this requirement is not a problem because the time history of the integrals can be easily differentiated numerically. If the observer is moving with respect to the fluid, as in the case of a wind-tunnel test, the situation becomes more complicated because the formulation requires the observer to be stationary during the evaluation of the integrals. Predictions with a moving observer are possible by adjusting the observer position at each time in the acoustic-pressure time history; however, three evaluations of the integrals are needed to perform a second-order centraldifference approximation to the time derivatives at each observer time. These extra integral evaluations are unnecessary if the time derivatives are taken inside the integrals analytically.

Although the derivation of a formulation with the time derivatives inside the integrals is not difficult, it is quite tedious. As a starting point, the integrals in equation (6) can be labeled conveniently as $I_{1}$, $I_{2}$, and $I_{3}$, respectively. Thus, equation (6) can be written as

$$
4 \pi p_{Q}^{\prime}(\vec{x}, t)=\frac{\partial^{2}}{\partial t^{2}}\left(I_{1}\right)+\frac{\partial}{\partial t}\left(I_{2}\right)+I_{3}
$$

Now, to take the observer time derivatives inside the integrals we use the relationship given by Farassat and Succi: ${ }^{18}$

$$
\left.\frac{\partial}{\partial t}\right|_{\vec{x}}=\left[\left.\frac{1}{1-M_{r}} \frac{\partial}{\partial \tau}\right|_{\vec{x}}\right]_{r e t}
$$

This result is obtained by using the definition $g=$ $\tau-t+r / c=0$, given the fact that $r$ is a function of source time $\tau$ through the source position variable $\vec{y}$. Two applications of relation (8) on $I_{1}$ yield

$$
\begin{aligned}
\frac{\partial^{2}}{\partial t^{2}}\left(I_{1}\right) & =\int_{f+=0}\left[K_{1} Q_{r r}\right]_{r e t} d S \\
& +\int_{f+=0}\left[K_{2} \frac{\partial Q_{r r}}{\partial \tau}\right]_{r e t} d S \\
& +\int_{f+=0}\left[K_{3} \frac{\partial^{2} Q_{r r}}{\partial \tau^{2}}\right]_{r e t} d S
\end{aligned}
$$

where $K_{1}, K_{2}$, and $K_{3}$ are defined as

$$
\begin{aligned}
K_{1} & =\frac{\frac{\partial^{2} M_{r}}{\partial \tau^{2}}}{c^{2} r\left(1-M_{r}\right)^{4}}+\frac{3\left(\frac{\partial M_{r}}{\partial \tau}\right)^{2}}{c^{2} r\left(1-M_{r}\right)^{5}} \\
& +\frac{\frac{\partial M_{r}}{\partial \tau}\left(1+2 M_{r}\right)}{c r^{2}\left(1-M_{r}\right)^{4}}+\frac{2 M_{r}^{2}}{r^{3}\left(1-M_{r}\right)^{3}}
\end{aligned}
$$

$$
K_{2}=\frac{3 \frac{\partial M_{r}}{\partial \tau}}{c^{2} r\left(1-M_{r}\right)^{4}}+\frac{2 M_{r}}{c r^{2}\left(1-M_{r}\right)^{3}}
$$

and

$$
K_{3}=\frac{1}{c^{2} r\left(1-M_{r}\right)^{3}}
$$

The time differentiation taken inside $I_{2}$ yields

$$
\begin{aligned}
\frac{\partial}{\partial t}\left(I_{2}\right) & =\int_{j+=0}\left[K_{4}\left(3 Q_{r r}-Q_{i i}\right)\right]_{r e t} d S \\
& +\int_{f+=0}\left[K_{5}\left(3 \frac{\partial Q_{r r}}{\partial \tau}-\frac{\partial Q_{i i}}{\partial \tau}\right)\right]_{r e t} d S
\end{aligned}
$$

with $K_{4}$ and $K_{5}$ defined as

$$
K_{4}=\frac{\frac{\partial M_{r}}{\partial \tau}}{c r^{2}\left(1-M_{r}\right)^{3}}+\frac{2 M_{r}}{r^{3}\left(1-M_{r}\right)^{2}}
$$

and

$$
K_{5}=\frac{1}{c r^{2}\left(1-M_{r}\right)^{2}}
$$

Terms $K_{1}$ through $K_{5}$ depend only on the kinematics of the source motion and have been determined by using relation (8) together with

$$
\frac{\partial r}{\partial \tau}=-c M_{r}
$$

The values of $K_{1}$ through $K_{5}$ can be evaluated by utilizing the definitions of the source-time derivatives of $M_{r}$, which are written as

$$
\frac{\partial M_{r}}{\partial \tau}=\dot{M}_{r}+\frac{c}{r}\left(M_{r}^{2}+M^{2}\right)
$$

and

$$
\begin{aligned}
\frac{\partial^{2} M_{r}}{\partial \tau^{2}} & =\ddot{M}_{r}+\frac{3 c}{r}\left(\dot{M}_{r}-M_{i} \dot{M}_{i}\right) \\
& +\frac{3 c^{2} M_{r}}{r^{2}}\left(M_{r}^{2}-M^{2}\right)^{2}
\end{aligned}
$$

These relations follow directly both from the fact that $\partial \vec{y} / \partial \tau=\vec{v}=c \vec{M}$ and from the definition of $r$.

The derivation would be complete at this point if the time-dependent values of $Q_{r r}$ and $Q_{i i}$ were given as input for each point in the source region. To compute $Q_{r r}$, however, requires knowledge of the observer position, which is unrelated to the flowfield calculations. For this reason and to take full advantage of the in-plane observer assumption, we assume that the input data do not require knowledge of the observer position. Hence, the determination of the source-time derivatives of $Q_{r r}$ and $Q_{i i}$ remains. 
By recalling that

$$
Q_{r r}=Q_{i j} \hat{r}_{i} \hat{r}_{j}
$$

and

$$
\frac{\partial \hat{r}_{i}}{\partial \tau}=\frac{c\left(\hat{r}_{i} M_{r}-M_{i}\right)}{r}
$$

we can easily to show that

$$
\begin{gathered}
\frac{\partial Q_{r r}}{\partial \tau}=\dot{Q}_{r r}+\frac{2 c\left(M_{r} Q_{r r}-Q_{M M}\right)}{r} \\
\frac{\partial^{2} Q_{r r}}{\partial \tau^{2}}=\ddot{Q}_{r r} \\
+\frac{2 c}{r}\left(\dot{M}_{r} Q_{r r}+2 M_{r} \dot{Q}_{r r}-2 \dot{Q}_{M r}-Q_{\dot{M} r}\right) \\
+\frac{2 c^{2}}{r^{2}}\left[Q_{M M}+\left(4 M_{r}^{2}-M^{2}\right) Q_{r r}-4 M_{r} Q_{M r}\right]
\end{gathered}
$$

and, by definition,

$$
\frac{\partial Q_{i i}}{\partial \tau}=\dot{Q}_{i i}
$$

The notation used here is defined in the Notation section; however, the reader should understand that the dot $\left(^{\circ}\right)$ implies source time differentiation on the variables shown with the dot. A dot on the main variable does not imply differentiation of any of the associated vectors implied by the subscripts. Subscripts other than $i$ and $j$ are a shorthand for the inner product of the main quantity with the vector represented by the subscript.

The final form, which is obtained by combining the previous results, becomes

$$
4 \pi p_{Q}^{\prime}(\vec{x}, t)=\int_{f+=0}\left[\frac{K_{r 1}}{c^{2} r}+\frac{K_{r 2}}{c r^{2}}+\frac{K_{r 3}}{r^{3}}\right]_{r e t} d S
$$

where

$$
\begin{aligned}
K_{r 1} & =\frac{\ddot{Q}_{r r}}{\left(1-M_{r}\right)^{3}}+\frac{\ddot{M}_{r} Q_{r r}+3 \dot{M}_{r} \dot{Q}_{r r}}{\left(1-M_{r}\right)^{4}} \\
& +\frac{3 \dot{M}_{r}^{2} Q_{r r}}{\left(1-M_{r}\right)^{5}} \\
K_{r 2} & =\frac{-\dot{Q}_{i i}}{\left(1-M_{r}\right)^{2}}-\frac{4 \dot{Q}_{M r}+2 Q_{\dot{M} r}+\dot{M}_{r} Q_{i i}}{\left(1-M_{r}\right)^{3}} \\
& +\frac{3\left[\left(1-M^{2}\right) \dot{Q}_{r r}-2 \dot{M}_{r} Q_{M r}-M_{i} \dot{M}_{i} Q_{r r}\right]}{\left(1-M_{r}\right)^{4}} \\
& +\frac{6 \dot{M}_{r}\left(1-M^{2}\right) Q_{r r}}{\left(1-M_{r}\right)^{5}}
\end{aligned}
$$

and

$$
\begin{aligned}
K_{r 3} & =\frac{2 Q_{M M}-\left(1-M^{2}\right) Q_{i i}}{\left(1-M_{r}\right)^{3}}-\frac{6\left(1-M^{2}\right) Q_{M r}}{\left(1-M_{r}\right)^{4}} \\
& +\frac{3\left(1-M^{2}\right)^{2} Q_{r r}}{\left(1-M_{r}\right)^{5}}
\end{aligned}
$$

Equation (17), together with the definitions of $K_{r 1}$, $K_{r 2}$, and $K_{r 3}$, will be referred to as formulation Q1A and is the main result of this paper. The designation Q1A is intended to parallel that of Farassat's formulation $1 \mathrm{~A},{ }^{17}$ in which the observer time derivative is taken analytically inside the thickness and loading integrals. Formulation Q1A does not require numerical time differentiation of the integrals and, as a retarded-time formulation, is well suited for subsonic source motion. Aside from the problem geometry, only the time-dependent value of $Q_{i j}$ is required as input.

Note that the definition of the Lighthill stress tensor $T_{i j}$, as well as that of $Q_{i j}$, requires that the fluid velocities be specified in an inertial reference frame fixed to the undisturbed medium. In this frame, the fluid velocities are perturbations about the stationary medium. Often, CFD solutions are computed in a body-fixed reference frame; hence, the motion of the body must be removed. Finally, remember that the vector $\vec{M}$ (and all related quantities such as $M_{r}$ ) is the source velocity rather than the fluid velocity.

The remainder of this paper will discuss the numerical implementation of equation (17) and present some initial predictions.

\section{Numerical Implementation}

The numerical calculation of the quadrupole noise has been divided into two stages: a preprocessing stage in which the integration of the Lighthill stress tensor in the normal direction, indicated in equation (3), is carried out, and an evaluation stage in which the quadrupole contribution to the acoustic pressure specified in equation (17) is determined. Both the preprocessor and the acoustic calculation are described in this section. More information on the program details can be found in reference 14 .

\section{Preprocessor}

Although the evaluation of $Q_{i j}$ can be performed independently of the observer position and the retarded time, the preprocessor must read in the CFD solution, interpolate the solution at the necessary quadrature locations, and then perform the numerical quadrature in the direction normal to the rotor disk. The preprocessor needs knowledge of both the CFD grid topology and the solution format. In the initial implementation used for this work, the inter- 
polation of the CFD data is two dimensional and is done one radial station at a time. For a given radial station, data are interpolated to quadrature points needed for composite Gauss-Legendre integration, on lines normal to the rotor plane. The lines are uniformly distributed in the chordwise direction. A two-dimensional linear least-squares interpolation is used to interpolate the density, momentum, and energy at each quadrature point. The Lighthill stress tensor $T_{i j}$ is evaluated with the interpolated data. The value of $Q_{i j}$ on $f^{+}=0$ is determined at each chordwise location before moving to the next radial station. The entire process is repeated for each source time and is stored as input to the acoustic calculation stage.

\section{Noise Calculation}

The quadrupole noise calculation indicated in equation (17) is carried out in a modified version of WOPWOP, ${ }^{17}$ called WOPWOP+. The quadrupole noise calculation is distinct from but parallels that of thickness and loading noise in the WOPWOP code, so the discussion here will be brief. The WOPWOP+ code used in this paper is essentially the same as that used in reference 14, with two exceptions. First, the code was modified to use unsteady quadrupole source loading. Second, formulation Q1A was implemented to enable forward-flight wind-tunnel predictions.

One unique aspect of the quadrupole noise calculation is that the integration surface extends ahead of the leading edge, behind the trailing edge, and off the rotor-blade tip. Because of this difference in the integration surface, the quadrupole noise calculation is a separate subroutine in WOPWOP+. The panels that extend off the tip can be a problem for a retarded-time formulation because rotating panels move supersonically if the radius of rotation is great enough. Rather than implement specialized code logic to handle the singular integrals caused by supersonic panel motion, only panels with subsonic speeds are included in the present calculations.

Because the CFD solutions used in this work are output in a coordinate system that is always aligned with the rotor blade, coordinate transformations are used to transform $Q_{i j}$ into the ground fixed frame used for all acoustic calculations. The numerical integration over each panel is carried out for all observer times before proceeding to the next source panel to reduce the retarded-time differences between subsequent evaluations. Unlike the thickness and loading algorithm in WOPWOP, which allows the user to select the number of computation panels, the quadrupole calculation in WOPWOP+ uses the computational grid from the preprocessor without modification. Gauss-Legendre quadrature is used in both the chordwise and radial directions for the numerical integration over each panel. The retarded time is determined by using the modified false position method.

An adaptive numerical integration scheme is used to compute the contribution of the quadrupole noise from each panel. The motivation for using an adaptive scheme is that, although the quadrupole source strength may vary essentially linearly over a panel, experience has shown that when the retarded time is properly taken into account a larger number of quadrature points is needed if the effective area (i.e., the emission area $\Delta \Sigma$, also called the acoustic planform) of the panel is large. The panel area, panel velocity, and observer position all determine the acoustic planform of the panel. When the acoustic planform of the panel is large, more quadrature points are expected to be needed to perform the integration over the panel because the integrated function may have more variation. An adaptation parameter given by

$$
N\left(M_{r}, A\right) \propto \frac{A}{\left|1-M_{r}\right|}
$$

where $M_{r}$ is the Mach number of the panel in the radiation direction and $A$ is the physical area of the panel, is used to determine the number of quadrature points for a particular panel; $N$ is scaled such that $1 \leq N \leq 20$. As $M_{r}$ approaches unity or if the area of the panel is large, the adaptation parameter becomes large. Similarly, if $M_{r}$ is small or the area of the panel is small, then the adaptation parameter becomes small. For this paper, only the number of points in the chordwise direction has been varied because little difference is found when more points in the radial direction are used.

\section{$\underline{\text { Sample Predictions }}$}

In this section, two representative calculations are performed to demonstrate the new formulation and to provide some indication of the efficiency and robustness of the new quadrupole prediction method. Predicted acoustic-pressure time histories will be compared with measured data for both hover and forward-flight conditions.

\section{Hover}

A model scale rotor test, conducted by Boxwell et al. ${ }^{19}$ in 1978 and later repeated by Purcell $^{20}$ in 1988, was selected for validation of the present theory and code. Both experiments were designed specifically to measure HSI noise generated by a nonlifting helicopter rotor in hover. The rotor was a 


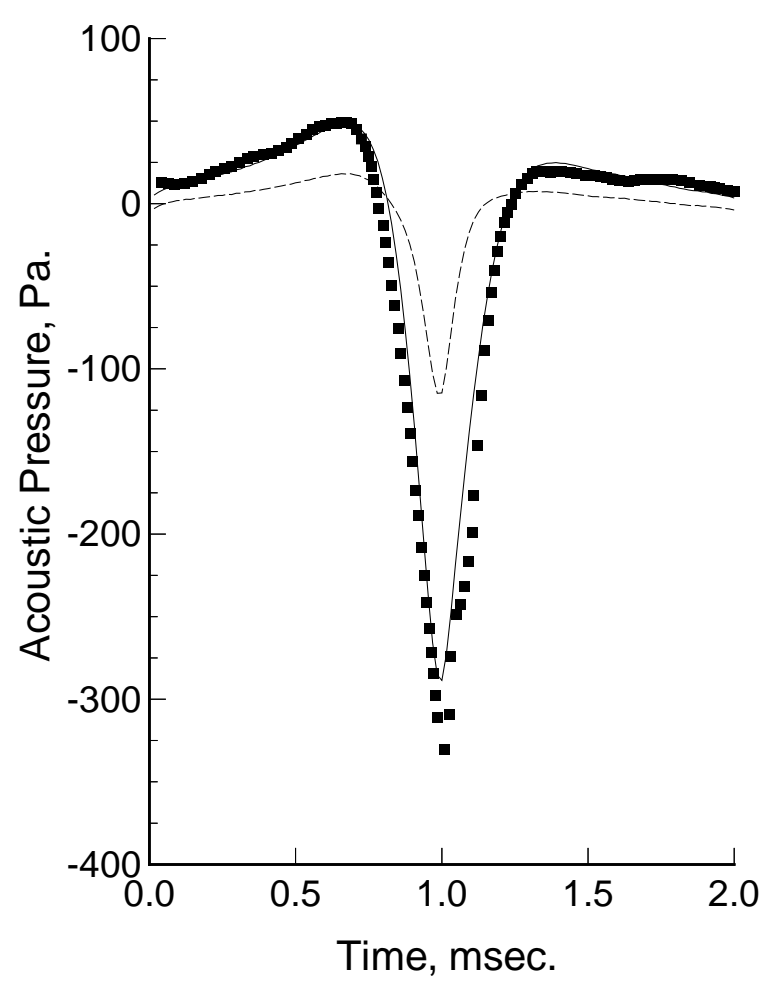

Figure 3. UH-1H model rotor operating in hover with $M_{H}=0.88$. experimental data; — predicted acoustic pressure; - - - quadrupole component of predicted acoustic pressure.

one-seventh scale model of a $\mathrm{UH}-1 \mathrm{H}$ main rotor with straight, untwisted blades. The model rotor utilized an NACA 0012 airfoil section. The rotor radius $R$ was $1.045 \mathrm{~m}$ with a chord of $7.62 \mathrm{~cm}$. The model was run at several high-speed hover conditions with low thrust. Comparisons of measured and predicted acoustic pressure are made for an in-plane microphone located $3.09 R$ from the rotor tip.

For the hover noise calculation, an Euler solution reported by Baeder et al. ${ }^{21}$ was used as input. The Euler calculations were performed on a $\mathrm{C}-\mathrm{H}$ grid; only the lower half of the grid was used in the calculations by taking advantage of the symmetry of the problem. The Euler calculations required approximately 80 min of CPU time on a Cray Y-MP. Details of the Euler calculations can be found in references 21 and 22 .

In figure 3 , the predicted quadrupole component and the total acoustic pressure are compared to the experimental data for a tip Mach number $M_{H}=$ 0.88 . For this case, the flow around the UH-1H rotor blade is transonic but not delocalized; neverthe- less, the quadrupole contribution to the total signal is substantial. The good agreement between the current prediction and the experimental data is representative of HSI noise predictions with this new quadrupole formulation when the rotor speed is below the delocalization Mach number. Although the result here is a new computation using formulation Q1A, this result is indistinguishable from the previous calculation by Brentner and Holland, ${ }^{14}$ which used formulation Q1. For this preliminary calculation, approximately 6 sec of CPU time on a scientific workstation was required for the preprocessor execution, and an additional $40 \mathrm{sec}$ was needed to run the combined thickness, loading, and quadrupole noise prediction.

\section{Forward Flight}

To demonstrate the forward-flight capability of the new formulation and code, a comparison of predicted and measured results for a four-blade swepttip rotor tested in the Duits-Nederslandse Windtunnel (DNW) was made. For this comparison, a microphone located in the rotor plane at a rotor azimuth of $\psi=150^{\circ}$ was utilized. The model rotor was operated at an advance ratio $\mu=0.32$ and an advancing tip Mach number $M_{A T}=0.933$. The experiment is described in the report by Visintainer et al. ${ }^{23}$

For this prediction, the full potential solver FPRBVI ${ }^{24}$ was used to compute the unsteady flow field around the rotor. For this developmental calculation, the FPRBVI code was modified to store a solution file at every degree of rotor azimuth. These files were then read by the preprocessor program to compute the time-dependent values of $Q_{i j}$. Manipulation of the three-dimensional time-dependent data was computationally demanding in terms of CPU time and storage. For this case, approximately 1100 $\mathrm{CPU}$ sec on a scientific workstation was required by the preprocessor. Both computer time (by reducing input and output requirements) and storage could be reduced significantly if the preprocessor was included as a data post-processing subroutine in the CFD code.

The results of the forward-flight prediction are shown in figure 4. Again, the experimental data are compared with the predicted acoustic pressure; the quadrupole contribution is also shown to indicate its relative magnitude. Although the $\mathrm{CFD}$ calculation used a rather coarse grid, the agreement is good. Further work is necessary, however, to determine whether the CFD solution is fully converged and to determine the sensitivity of the quadrupole prediction to various input parameters. This developmen- 


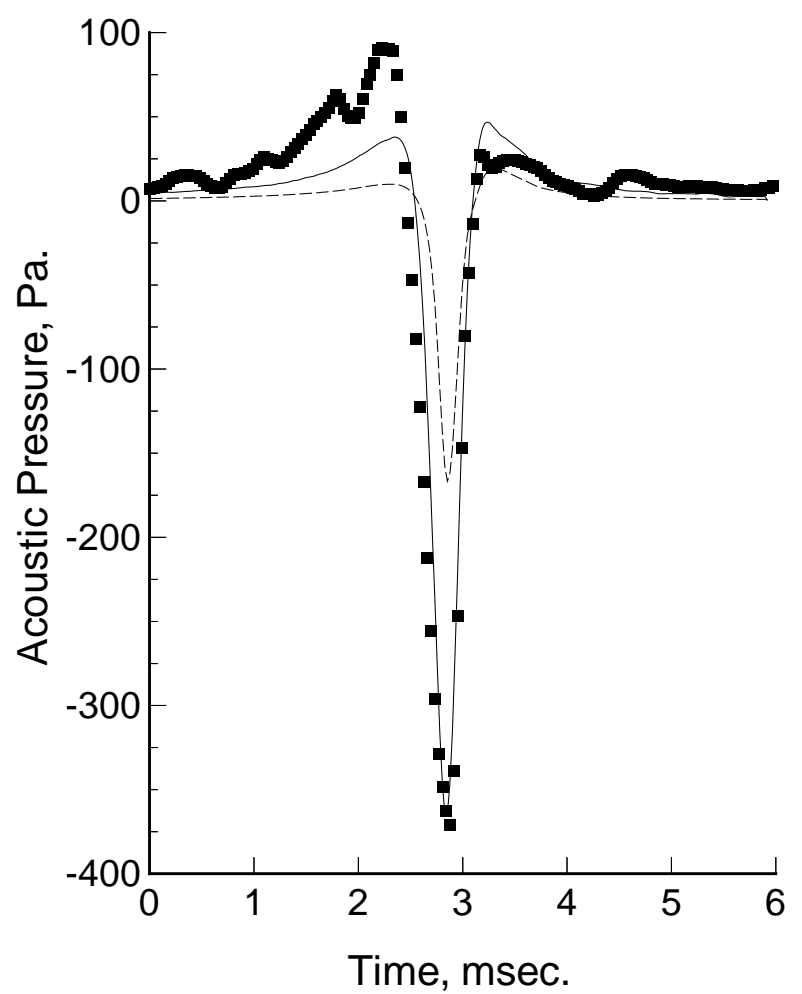

Figure 4. Contemporary design, four-blade model rotor operating in forward flight; $\mu=0.32$ and $M_{A T}=0.933$. experimental data; — predicted acoustic pressure; - - quadrupole component of predicted acoustic pressure.

tal calculation required approximately $190 \mathrm{CPU}$ sec on a scientific workstation.

\section{Concluding Remarks}

The new far-field quadrupole noise formulation presented in this paper provides an efficient method for computing high-speed impulsive (HSI) noise. The method is efficient in the sense that the volume integration normally associated with the Ffowcs Williams-Hawkings ( $\mathrm{FW}-\mathrm{H})$ quadrupole has been reduced to a surface integration of the same form as thickness and loading noise. Taking the time derivatives inside the integrals also improves the efficiency of the numerical method by avoiding extra integral evaluations required by numerical time differentiation. Even though the code has not been optimized, the acoustic predictions are already relatively fast for both hover and forward-flight predictions.

The new formulation should also prove to be robust. Brentner and Holland ${ }^{14}$ have demonstrated that the far-field approximation gives the correct signal amplitude, even for high-speed cases in which the present retarded-time formulation does not strictly apply. In these cases, supersonic quadrupole sources are ignored in WOPWOP+; the signal shape suffers and is incorrect. This graceful failure is characteristic of the robust nature of retarded-time formulations. The efficiency and robustness of this new quadrupole formulation make the acoustic analogy an attractive option for the prediction of HSI noise and will help to maintain the usefulness of rotor noise prediction codes based on the $\mathrm{FW}-\mathrm{H}$ equation.

\section{Acknowledgments}

The author would like to express appreciation to Dr. J. D. Baeder for providing the Euler calculation used as input for the hover prediction, and C. L. Burley and D. S. Prichard for their assistance with the experimental data and setting up the FPR calculation used in the forward-flight comparison. The author wishes to acknowledge that P. C. Holland assisted in early stages of this work.

\section{$\underline{\text { References }}$}

1. Ffowcs Williams, J. E. and Hawkings, D. L., "Sound Generated by Turbulence and Surfaces in Arbitrary Motion," Philosophical Transactions of the Royal Society, Vol. A264, 1969, pp. $321-342$.

2. Lighthill, M. J., "On Sound Generated Aerodynamically. I: General Theory," Proceedings of the Royal Society, Vol. A221, 1952, pp. 564587.

3. Lighthill, M. J., "On Sound Generated Aerodynamically. II: Turbulence as a Source of Sound," Proceedings of the Royal Society, Vol. A222, 1954, pp. 1-32.

4. Yu, Y. H., Caradonna, F. X., and Schmitz, F. H., "The Influence of the Transonic Flow Field on High-Speed Helicopter Impulsive Noise," Fourth European Rotorcraft and Powered Lift Aircraft Forum, paper 58, 1978.

5. Hanson, D. B. and Fink, M. R., "The Importance of Quadrupole Sources in Prediction of Transonic Tip Speed Propeller Noise," Journal of Sound and Vibration, Vol. 62, 1979, pp. 1938 .

6. Schultz, K. J. and Splettstoesser, W. R., "Prediction of Helicopter Rotor Impulsive Noise Using Measured Blade Pressure," American Helicopter Society 43rd Annual Forum, St. Louis, MO, 1987. 
7. Schultz, K. J., Lohman, D., Lieser, J. A., and Pahlke, K. D., "Aeroacoustic Calculation of Helicopter Rotors at DLR," AGARD Fluid Dynamics Panel Symposium on Aerodynamics and Aeroacoustics of Rotorcraft, paper 29, 1994.

8. Ianniello, S. and De Bernardis, E., "Calculation of High-Speed Noise from Helicopter Rotor Using Different Descriptions of Quadrupole Source," AGARD Fluid Dynamics Panel Symposium on Aerodynamics and Aeroacoustics of Rotorcraft, paper 27, 1994.

9. Prieur, J., "Calculation of Transonic Rotor Noise Using a Frequency Domain Formulation," AIAA Paper 86-1901, 1986.

10. Prieur, J., Costes, M., and Baeder, J. D., "Aerodynamic and Acoustic Calculations of Transonic Nonlifting Hovering Rotors," AHS/RAeS International Technical Specialists' Meeting on Rotorcraft Acoustics and Rotor Fluid Dynamics, Philadelphia, PA, 1991.

11. Farassat, F., "Quadrupole Source in Prediction of Noise of Rotating Blades-A New Source Description," AIAA Paper 87-2675, 1987.

12. Farassat, F. and Myers, M. K., "An Analysis of the Quadrupole Noise Source of High Speed Rotating Blades," in Computational Acoustics 2 (D. Lee, A. Cakmak, and R. Vichnevetsky, eds.), Amsterdam: North-Holland, 1990, pp. $227-240$.

13. Tadghighi, H., Holz, R., Farassat, F., and Lee, Y. J., "Development of a Shock Noise Prediction Code for High-Speed Helicopters," American Helicopter Society 47th Annual Forum, Phoenix, AZ, 1991.

14. Brentner, K. S. and Holland, P. C., "An Efficient and Robust Method for Computing Quadrupole Noise," American Helicopter Society 1995 Aeromechanics Specialist Conference, Bridgeport, CT, 1995.

15. Farassat, F. and Brentner, K. S., "The Uses and Abuses of the Acoustic Analogy in Helicopter Rotor Noise Prediction," Journal of the American Helicopter Society, Vol. 33, 1988, pp. $29-36$.

16. Farassat, F., "Theory of Noise Generation from Moving Bodies with an Application to Helicopter Rotors," NASA TR R-451, 1975.
17. Brentner, K. S., "Prediction of Helicopter Rotor Noise-A Computer Program Incorporating Realistic Blade Motions and Advanced Formulation," NASA TM-87721, 1986.

18. Farassat, F. and Succi, G. P., "The Prediction of Helicopter Discrete Frequency Noise," Vertica, Vol. 7, No. 4, 1983, pp. 309-320.

19. Boxwell, D. A., Yu, Y. H., and Schmitz, F. H., "Hovering Impulsive Noise: Some Measured and Calculated Results," NASA CP-2052, 1978. Also in Vertica, Vol. 3, No. 1, 1979, pp. 35-45.

20. Purcell, T. W., "CFD and Transonic Helicopter Sound," Fourteenth European Rotorcraft Forum, paper 2, 1988.

21. Baeder, J. D., Gallman, J. M., and Yu, Y. H., "A Computational Study of Aeroacoustics of Rotors in Hover," American Helicopter Society 49th Annual Forum, St. Louis, MO, 1993.

22. Baeder, J. D., "Euler Solutions to Nonlinear Acoustics of Non-Lifting Rotor Blades," AHS/RAeS International Technical Specialists' Meeting on Rotorcraft Acoustics and Rotor Fluid Dynamics, Philadelphia, PA, 1991.

23. Visintainer, J. A., Marcolini, M. A., Burley, C. L., and Liu, S. R., "Acoustic Predictions Using Measured Pressures from a Model Rotor in the DNW," Journal of the American Helicopter Society, Vol. 38, No. 3, 1993, pp. 35-44.

24. Burley, C. L. and Tadghighi, H., "Importance of High Accuracy Blade Motion and Airloads Predictions in Acoustic Analysis," American Helicopter Society 50th Annual Forum, Washington, D. C., 1994. 